\title{
Medievalista
}

Online

$29 \mid 2021$

Número 29

Une lecture zoopoétique du Chevalier au Lion de Chrétien de Troyes

\section{Bestiaire en marge}

Bestiary in the margins. A zoopoetic reading of Le Chevalier au Lion de

Chrétien de Troyes

\section{Cristina Álvares}

\section{OpenEdition}

Journals

\section{Édition électronique}

URL : https://journals.openedition.org/medievalista/3918

DOI : 10.4000/medievalista.3918

ISSN : 1646-740X

\section{Éditeur}

Instituto de Estudos Medievais - FCSH-UNL

\section{Édition imprimée}

Pagination : 277-296

\section{Référence électronique}

Cristina Álvares, «Bestiaire en marge », Medievalista [En ligne], 29 | 2021, mis en ligne le 01 janvier 2021, consulté le 11 juin 2021. URL : http://journals.openedition.org/medievalista/3918 ; DOI : https:// doi.org/10.4000/medievalista.3918

Ce document a été généré automatiquement le 11 juin 2021.

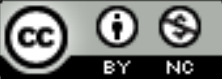

Mediavalista está licenciado com uma Licença Creative Commons - Atribuição-NãoComercial 4.0 Internacional. 
Une lecture zoopoétique du Chevalier au Lion de Chrétien de Troyes

\title{
Bestiaire en marge
}

\author{
Bestiary in the margins. A zoopoetic reading of Le Chevalier au Lion de
}

Chrétien de Troyes

Cristina Álvares

\section{NOTE DE L'ÉDITEUR}

Data recepção do artigo / Received for publication: 17 de Dezembro de 2019

Data aceitação do artigo / Accepted in revised form: 5 de Junho de 2020

Pensée symbolique et liminalités

2 Dans Par-delà nature et culture, Philippe Descola élabore une nouvelle théorie anthropologique visant à dépasser le dualisme nature-culture considéré obsolète en raison notamment de l'artificialisation croissante de la nature par la technologie. Il distingue quatre ontologies ou modalités de configuration des relations entre humains et non-humains: l'animisme, le totémisme, le naturalisme et l'analogisme. L'analogisme est exemplifié, entre autres, par la culture occidentale du Moyen Âge. En effet, la vision du monde médiévale distribue les êtres et les espèces en des catégories (l'humain, le divin, l'animal, le végétal, le minéral) séparées par des écarts ou frontières ontologiques qui les hiérarchisent au sein de la sphère de la Création ${ }^{1}$. Anthropologiquement constitutive, la frontière séparant humain et animal établit une différence irréductible entre les deux règnes dont la relation ne serait que de distance si la cosmovision symbolique ne tissait pas entre l'homme et l'animal un réseau de correspondances analogiques ${ }^{2}$ qui rapproche et associe les deux catégories. À ce propos, Pierre-Olivier Dittmar écrit:

“C'est ainsi que le Physiologos (IIe siècle), les différents bestiaires et les encyclopédies, mettent en parallèle les mondes humains et animaux, énumérant les similitudes de rapports entre les deux séries, et actant par le même mouvement la distance irréductible qui sépare les deux règnes"3. 
La ressemblance ne supprime pas la différence entre humain et animal, au contraire elle la présuppose. Lorsqu'il y a suppression, lorsque le rapprochement les rassemble dans la métamorphose ou l'hybridation, on a affaire à la transgression. C'est la thèse soutenue par les auteurs de Image et transgression au Moyen Âge ${ }^{4}$ : quand une image mélange et amalgame des espèces pour produire un monstre, elle transgresse les frontières ontologiques qui soutiennent l'ordre de la Création. L'image transgressive signale une crise, un désordre, une aberration sans place et sans nom, au comble de la dégradation post-Chute. Elle est donc un contre-modèle à valeur morale, comme le prouve la prolifération des hybrides à la fin du Moyen Âge culminant dans la peinture de Jérôme Bosch.

Nous comprenons ainsi que la figuration métonymique qui structure les images transgressives est problématique pour l'analogisme aussi bien explicite (comparaison) qu'implicite (métaphore). La mise en parallèle des sphères humaine et animale se déploie sur la différence anthropologique, alors que la contiguiité métonymique aplatit la hiérarchie et produit des zones de voisinage, de continuité et d'intersection entre espaces et identités hétérogènes. Là où la similitude garde la frontière ontologique, la métonymie la change en seuil, passage, devenir. Là où la métaphore fait résonner l'harmonie de la Création, la métonymie dissone. Certes, sa portée transgressive est réabsorbée dans la pensée symbolique qui l'investit d'une signification morale (le péché et sa punition). Il n'en reste pas moins que la métonymie introduit à l'intérieur du système des correspondances analogiques entre des êtres, une logique autre dont la dynamique (circulation, changement, devenir) ébranle l'ordre. Une tension est créée qui détotalise et dialectise la pensée symbolique. Il a beau valorisé l'ordre, l'essence, le statut, l'Occident médiéval n'est pas pour autant un ordre social et culturel homogène et statique, loin de là. C'est la thèse que Carlos Carreto soutient dans une étude récente ${ }^{5}$ où, à propos des articulations entre les études médiévales et les études globales (Global Studies), il analyse plusieurs dynamiques de globalisation dans le monde médiéval qui s'auto-perçoit typiquement comme un globe contenant des globes (le topos de l'harmonie des sphères). Vue comme une aventure urbaine qui émerge aux VIIIe et IXe siècles et s'intensifie au XIIe siècle au sein de la Chrétienté latine, formation transfrontalière s'il en est, la globalisation médiévale entraîne des gens, des objets, des idées, dans le tourbillon d'une mutation mise à l'enseigne du marchand et de la circulation: intensification des échanges commerciaux à des échelles de plus en plus larges, valorisation postféodale de la monnaie et des biens meubles ${ }^{6}$. Sans épuiser la globalisation médiévale (car sa composante (inter)médiale passe par la textualisation des institutions et de la culture ${ }^{7}$ dont la figure majeure est le clerc), la circulation de marchandises et de capitaux modélise toute sorte de déplacements et d'itinérances plus ou moins erratiques (voyages, pèlerinages, croisades, itinérances jongleuresques). Ces dynamiques témoignent d'une vitalité qui n'a pas exactement dé-sphérisé le globe mais qui a érodé la consistance de sa morphologie et préparé l'aplatissement de la sphère cosmique en sphère terrestre - aplatissement qui constitue, selon Peter Sloterdijk, l'événement fondamental des temps modernes8.

5 La tension dialectique entre ordre et dérive ou entre forme sphérique et tourbillon informe ou encore entre globe et globalisation, travaille en profondeur le Moyen Âge occidental, sa pensée et ses créations artistiques et littéraires. Une telle tension ouvre la pensée symbolique à un champ d'altérité et cette ouverture a un effet pluralisant, diversifiant et relativisant sur l'ontologie analogiste: il y a d'autres modes 
d'appréhender la réalité, de percevoir et de représenter les relations entre les êtres et les catégories, notamment entre humain et animal. De ces modes autres la littérature en langue vulgaire, surtout le roman, est une forme majeure. L'accès de la langue vulgaire au statut littéraire, mouvement décisif de formation des modernes littératures européennes, a investi de prestige social et culturel un vaste segment de l'imaginaire décalé par rapport à la culture latine de l'Église et à sa vérité théologique ${ }^{9}$. Cet imaginaire riche et diversifié, remontant à différentes origines préchrétiennes (grécoromaines, celtiques, germaniques), existait depuis longtemps, préservé dans des textes latins mais surtout dans les traditions orales, méprisées en tant que telles, et ce sont les clercs qui, en écrivant ces matières en langue vulgaire, leur ont donné, ainsi qu'à la langue (qui cesse d'être vulgaire), ses lettres de noblesse. Les fictions (carolingiennes, arthuriennes, antiquisantes) (ré)inventées au long du XIIe siècles par les clercs en roman, en particulier celles qui forment le genre moderne du roman - genre ainsi nommé en raison de sa genèse dans la langue vulgaire -, composent une poétique vernaculaire qui constitue un mode autonome d'appréhender la réalité ${ }^{10}$. La pensée qui les structure n'est pas symbolique ou ne l'est que partiellement ou occasionnellement. La figuration métonymique défie et déjoue les retentissements métaphoriques de l'harmonie des sphères moyennant la production de zones liminaires où des entités hétérogènes se touchent, se transmutent, se contaminent. Dans une formule empruntée à Lacan, nous dirons que la pensée médiévale n'est pas toute symbolique et, avec Armand Strubel, que la pensée symbolique était au Moyen Âge en voie d'épuisement ${ }^{11}$ ou en dysfonctionnement. La coexistence et l'interaction des deux voies majeures de figuration (sinon d'autres) doivent être soulignées contre le préjugé tenace, entretenu par la culture populaire et médiatique contemporaine, d'un Moyen Âge monolithique, homogène et atavique, à mentalité symbolique hégémonique, porté par une religion dualiste raisonnant sur le seul critère de l'opposition principielle de la chair et de l'esprit. C'est d'abord ignorer l'existence d'un imaginaire médiéval laïque; c'est ensuite ignorer que le christianisme n'est pas et n'a jamais été une religion dualiste en raison du dogme central de l'Incarnation, que le dualisme se trouvait du côté des hérésies que l'Eglise combattait et que le dogme de l'Incarnation est à la racine du cadre épistémologique où s'est développé la pensée scientifique ${ }^{12}$. Par ailleurs, les accusations adressées à la tradition judéo-chrétienne en ce qui concerne la légitimation de la maltraitance des animaux butent contre le franciscanisme qui lui-même se fonde du texte biblique ${ }^{13}$.

6 Les bestiaires, ouvrages didactiques qui décrivent des animaux réels ou légendaires comme allégories de formes et de fonctions humaines, constituent typiquement le genre le plus représentatif de la configuration analogique de la relation humain-animal propre à la pensée symbolique. Dans son Bestiaire, Pierre de Beauvais décrit certaines propriétés du lion dont la dernière est celle-ci:

"L'homme possède une partie de la nature du lion car celui-ci ne se mettra pas en colère à moins d'être blessé (...): il épargne les bêtes faibles et laisse aller en paix les petits animaux, et il ne tue aucun homme à moins d'y être poussé par une très grande faim. Les hommes de haut rang doivent conserver présent à l'esprit cet exemple de miséricorde, et épargner les pauvres et les faibles"14.

7 Le lion est ici la métaphore d'un trait humain: la miséricorde comme limite éthique à la violence de la prédation. La représentation anthropomorphique de l'animal le réduit à une image abstraite, en l'occurrence une allégorie de la miséricorde humaine, et cette abstraction évince son épaisseur réelle d'être vivant. 

de l'animal turn, se propose de lire les textes littéraires à partir du postulat fondamental selon lequel le statut ontologique-linguistique des animaux dans la littérature n'est pas celui de métaphores des êtres humains mais des êtres vivants dotés d'une puissance d'expressivité ${ }^{15}$. La zoopoétique, qui pense la création littéraire comme l'expression d'une poétique primordiale du vivant et l'institution d'une relation ontologique entre l'écriture et le vivant, s'intéresse moins aux images qu'au réel des animaux, pour autant que

"la littérature nous fait entrapercevoir les modes d'être diversifiés des bêtes, cellesci partageant avec nous non seulement une origine biologique commune mais une relation universelle des animés au monde - la même archè, bien que perçue et instituée selon des schémas corporels et des modalités sonsori-motrices souvent radicalement différents" ${ }^{16}$.

10 La sphère des vivants ("la même archè") est ici un espace partagé dans lequel les différences ne s'ordonnent pas hiérarchiquement mais se déploient sur l'étendue de la Terre. Cette morphologie horizontale de la biosphère est plus favorable aux procédés métonymiques qu'aux procédés métaphoriques. Aussi le projet zoopoétique nous invite-t-il à une relecture des œuvres du passé qui mette l'accent sur les "déplacements et des déconstructions stylistiques qui défigurent nos images humaines, trop humaines, des vivants". ${ }^{17}$ Ceci implique de considérer le réel des bêtes au-delà ou en-deçà du statut de reflet anthropomorphisé. Le terme réel réunit deux significations: un animal est un être de chair et de sang, un être singulier (pas une catégorie); un animal est un être de fuite qui toujours se dérobe à la capture sémantique.

11 Nous proposons une lecture zoopoétique du Chevalier au Lion de Chrétien de Troyes. Voici un roman des années 1180 dont le titre, mettant l'homme et l'animal côté à côte, amorce une figuration équivoque entre métaphore et métonymie. La tradition herméneutique et critique en français de ce roman penche pour la signification symbolique du lion ${ }^{18}$, alors que la tradition anglo-saxonne souligne l'irréductibilité du lion à une image ${ }^{19}$. Nous pensons qu'une approche zoopoétique pourra éclairer à nouveaux frais la présence du fauve à côté d'Yvain dans un roman qui pose dramatiquement la question de la frontière entre humain et animal en trois moments critiques: la rencontre de Calogrenant avec le bouvier, la folie d'Yvain qui coïncide avec son devenir-animal et, finalement, son amitié avec le lion au long de la seconde partie $\mathrm{du}$ roman. On décerne dans l'histoire du Chevalier au Lion une autre pensée des frontières ontologiques qui déplace et conteste la frontière majeure de l'ordre de la Création en figurant sa souplesse et perméabilité sous plusieurs modalités. Avec ce roman, Chrétien de Troyes a thématisé la relation humain-animal sous les figures liminaires de la rencontre, du passage, de la coprésence et de l'entraide. Chrétien fait ainsi partie des écrivains "passeurs d'entremondes" ${ }^{20}$, a fortiori parce que ces zones de bord où les sphères humaine et animale s'ouvrent et se touchent sont parcourues de "sollicitude infinie" ${ }^{21}$ : sollicitude de l'ermite envers le fou, sollicitude qu'échangent Yvain et le lion, Yvain sauvant le lion de l'étreinte du serpent et prenant soin de lui après les violents combats qui le malmènent; et le lion veillant le sommeil de son maître et compagnon et participant aux combats pour le protéger. Déjà dans les bestiaires, le lion est symbole d'un mode de sollicitude qui est la miséricorde. Dans le roman de Chrétien, la générosité, le soin, le souci, l'attention, l'affection sont des variations de la sollicitude, valeur axiale de la tradition judéo-chrétienne (la sollicitude 
infinie de Dieu pour ses créatures, l'amour du prochain, la charité), réactivée aujourd'hui dans nos sociétés dramatiquement confrontées à la vulnérabilité et à l'interdépendance des vivants (philosophie, politique, éthique du soin). Calogrenant. Énoncé à la première personne par un narrateur-personnage secondaire, ce récit fonctionne comme le déclencheur de l'aventure d'Yvain dans la mesure où il raconte une aventure ratée que le protagoniste devra accomplir. Après avoir quitté le vavasseur qui l'avait accueilli, Calogrenant arrive dans un essart où se battent férocement des taureaux sauvages gardés par un vilain :

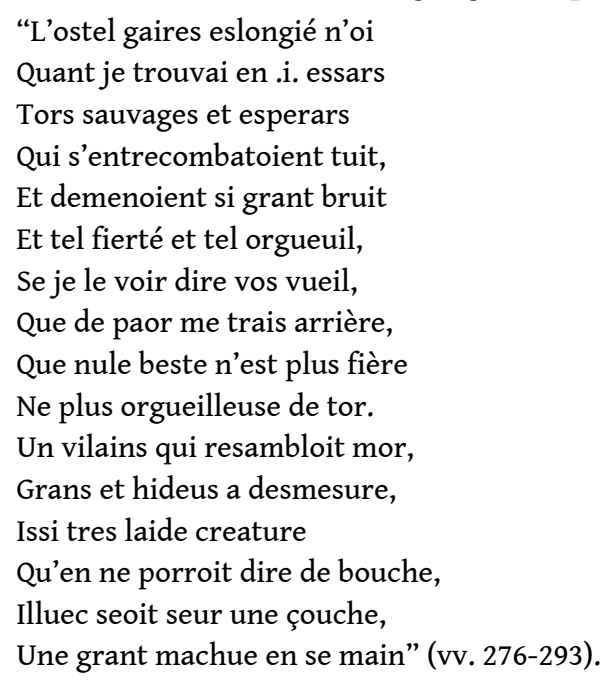

14 Ce personnage est décrit comme un patchwork de parties du corps de différentes espèces animales: oreilles d'éléphant, yeux de chouette, nez de chat, bouche de loup, dents de sanglier, etc. Le procédé métonymique produit une créature hétéroclite que Calogrenant ne sait pas situer par rapport à la frontière ontologique. En le supposant privé de langage, il essaye quand même une approche par la parole et à la question du chevalier qui lui demande quel genre de créature il est, le vilain répond simplement qu'il est un homme qui garde des bêtes. Élever des animaux est une activité humaine qui a la domestication comme cadre et condition, pourtant, les animaux élevés ici ne sont pas domestiques, mais sauvages et féroces. De plus, et Calogrenant s'en étonne, ils ne sont ni attachés ni enfermés. À ce que le vilain répond qu'il les maîtrise par la force brute de l'étreinte à plein corps:

"Car quant j'en puis l'une tenir,

Si le destraing par mi le cors,

Ad poins que j'ai et durs et fors,

Que les autres de paour tramblent,

Et tout en viron moi s'asamblent

Aussi com pour merchi crïer " (vv. 344-349)

Le pouvoir qu'il a sur les bêtes n'emploie donc pas les techniques d'apprivoisement inventées par les hommes dans la préhistoire, mais la supériorité de la force physique de cet homme qui est en fait un suranimal. Aussi bien le gardien des bêtes que les bêtes gardées se soustraient à la domestication et semblent renvoyer à une phase de l'évolution humaine antérieure au néolithique, comme l'indique le fait que le bouvier ne se couvre pas de tissus de lin ou de laine mais de peaux écorchées de taureaux. Dans l'adhésion de la peau animale à la peau humaine passe l'identification de l'animal 
humain aux autres qu'il domine. Mais ce contact métonymique des peaux, effet du combat corps-à corps pendant lequel l'animal est dominé, marque aussi le trait qui singularise cet épisode: la disjonction de la domination et de la domestication pour autant que la domination ne s'exerce pas sur des animaux domestiques mais se traduit dans un paradoxal pastoralisme de bêtes sauvages gardées dans un paradoxal enclos ouvert (elles n'osent pas sortir de peur du gardien). Les peaux écorchées des taureaux dont le vilain se revêt épinglent son féroce pastoralisme à la chasse et au fond de prédation inter-animale d'où elle a émergé. Le vilain apparait ainsi comme un homme archaïque, proto- ou sous-humain entouré des animaux, fauve parmi les fauves, habitant des interstices de l'animalité humaine. Est-ce le regard courtois de Calogrenant, chevalier plein d'urbanité et de politesse, qui hyperbolise en animalité la laideur et la rusticité du paysan? Celui-ci est tellement simple et rude qu'il ne comprend pas qu'il a affaire à un chevalier. Quand deux hommes doutent que chacun a affaire à un être de la même espèce, cela est sans doute le signe que la distance sociale qui les sépare est si excessive qu'elle prend des allures ontologiques. Quoi qu'il en soi, cet hybride qu'est le vilain ne semble pas symboliser une quelconque dégradation ou contamination causée par le péché. Au contraire, il indique la direction, il oriente, il met Calogrenant, puis Yvain, sur la voie de l'aventure. Gardien des bêtes sauvages, le vilain est aussi le gardien d'un seuil que les chevaliers doivent franchir pour entrer en aventure. Giorgio Agamben pense que l'aventure est l'expérience ontologique fondamentale par laquelle le vivant devient humain, c'est-à-dire se subjectivise grâce à l'amour ${ }^{22}$. Franchir le seuil par lequel l'animal devient homme et l'homme redevient animal est, selon Agamben, l'aventure des aventures. Or, c'est ce seuil qu'Yvain franchira dans les deux sens.

Le cru et le cuit

17 La deuxième occurrence d'une liminalité humain-animal implique le héros et apparait sous les traits de la folie. Tout en accomplissant l'aventure de la Fontaine, Yvain réussit là où Calogrenant avait échoué: il vainc le gardien de la Fontaine et épouse sa veuve de qui il tombe éperdument amoureux. Mais une fois que le récit atteint cet état d'équilibre, de bonheur et de stabilité, survient une nouvelle déstabilisation qui ouvre la sphère conjugale et domestique pour en extraire le héros et le jeter dans l'errance chevaleresque avec Gauvain. Ce passage de l'espace privé à l'espace public dérape sur un déséquilibre de la balance entre l'amour et la chevalerie: absorbé dans son agenda chevaleresque, Yvain a oublié de rentrer dans le délai fixé par la Dame. Cet oubli fait éclater la bulle déjà ébréchée et déclenche la crise majeure du roman, simultanément conjugale, subjective et narrative (puisqu'elle opère la transition de la première à la deuxième partie du roman). L'amour perdu, Yvain sombre dans la folie sous le mode du (re)devenir-animal, plus précisément un (re)devenir-fauve. Plein de haine pour soimême, Yvain s'éloigne des barons et fuit dans la forêt où il se dépouille des dispositifs anthropologiques qui produisent "le propre de l'homme": la présence des autres ses semblables, les vêtements, la mémoire, le langage, la raison. Nu et sauvage, il devient un prédateur que "les bestes par le bois aguete/et lors ochist, et si menjue/la venaison trestoute crue" (vv. 2824-26). Yvain fou est Yvain dédomestiqué, un homme qui refait à rebours et en quelques minutes le parcours de l'évolution de l'espèce jusqu'à la prédation et à la carnivorie, jusqu'au cru. La folie est une involution qui déspécialise l'homme en le réduisant à la condition d'animal affamé. Ou peut-être le respécialise-telle en le faisant re(de)venir à la condition animale d'où il est issu? Issu et sorti grâce au 
long apprentissage de la chasse dont la sévère discipline a constitué le facteur d'autodomestication le plus puissant ${ }^{23}$.

Il semble qu'Yvain a quand même gardé la mémoire de quelque chose de spécifiquement humain car pendant sa fuite il a volé un arc et des flèches et il est légitime de supposer qu'il utilise cette technique cynégétique spécifique à l'espèce humaine. La prédation n'est donc pas entièrement animale, mais passe par la médiation d'un outil. Ceci suggère que la folie-prédation d'Yvain est un compromis du cru (la dévoration de la chair crue) et du cuit, plus précisément la chasse comme prédation réorganisée dans et par la technique, sublimée en culture, base de la forme de vie humaine, élevée à la condition d'art. Le fou est donc un hybride de prédateur humain et de prédateur animal, de chasseur et de dévorateur. C'est aussi un compromis entre près et loin: avec l'art et les flèches, il capture la proie de loin, à distance, ensuite il dévore la venaison directement, sans passer par la médiation du feu.

Dans la forêt il y a des ermites, les romans médiévaux en regorgent ${ }^{24}$. La rencontre d'Yvain avec l'ermite crée les conditions du passage du cru au cuit et constitue une première étape dans la récupération progressive de la raison. L'ermite, lui aussi un homme des marges, homme (à demi) sauvage qui a fui l'ordre social pour se retrouver seul avec Dieu dans la nature, est quelqu'un qui vit à la lisière de la forêt, là où le monde sauvage touche au monde cultivé. Car le saint homme apolis défriche et fait du pain d'orge:

"Puis ne passa .i. jour entiers,

Tant comme il fu en chele rage,

Quë aucune beste sauvage

Ne li aportast a son huis.

Ycheste vie mena puis,

Et li boins hom s'entremetoit

De l'escorchier et si metoit

Assés de la venison cuire.

Et li pains et l'iaue en la buire

Estoit tous jours seur la fenestre

Pour l'omme forsené repaistre

(...)

Et li boins hom estoit en paine

Des cuirs vendre, d'acheter pain

D'orge, ou de paille ou de grain" (vv. 2868-78; 2882-84)

Entre l'ermite charitable et le fou prédateur carnivore un échange sans paroles s'instaure au seuil de la cabane: le fou dépose la venaison à la porte et l'ermite la fait cuire ou rôtir, il va au marché vendre les peaux et acheter du pain et ainsi nourrir l'homme fou. Sont ainsi réactivés des circuits sociaux et commerciaux élémentaires, hors langage, au sein desquels Yvain, rétabli dans le cuit, démarre la récupération de sa condition humaine ${ }^{25}$.

Dans les deux épisodes commentés, celui du vilain et celui du fou, l'enjeu est la domestication, déclinée négativement: un homme domine des animaux non domestiqués sur un essart, c'est-à-dire un bout du monde sauvage gagné au monde cultivé, donc marquant un seuil ; un homme se dédomestique, et c'est aussi sur un essart, celui dont s'occupe l'ermite, que commence sa re-domestication, c'est-à-dire sa réinsertion dans le monde humain avec ses règles et institutions. L'essart forme un bord où humain et animal se rejoignent, s'échangent, passent l'un dans l'autre ${ }^{26}$. 
Franchir ce seuil engage la domestication, celle des animaux, celle des hommes (Yvain se dédomestique dans la folie, il se redomestique grâce à l'ermite).

Le lion, un signe qui saigne

La domestication est au cœur de la rencontre d'Yvain et du lion qui a lieu aussi sur un essart. Après avoir récupéré la raison grâce aux soins de la demoiselle de Norison et d'avoir victorieusement combattu pour la Dame qui avait procuré l'onguent guérisseur ${ }^{27}$, Yvain pensif s'en va par la forêt, lorsque, soudainement, encore une fois sur un essart, il voit quelque chose qui le sort de sa torpeur: un énorme serpent tient un lion par la queue et lui brûle les reins d'une flamme ardente. Scène invraisemblable, car il n'y a pas de lions en Bretagne et que les serpents ne crachent pas de feu, ce sont les dragons qui le font, cette merveille enclave un segment de bestiaire dans le roman. Pour choisir la bête à qui porter secours, le chevalier raisonne dans les termes anthropomorphiques des bestiaires: le serpent est enuious e felon, le lion est beste gentil et franche. L'attribution à chacun des animaux de propriétés morales opposées, relevant du bien et du mal, est une première différentiation de cet hybride, la seconde étant le coup d'épée qui sépare les deux bêtes. Ce geste diaïrétique a forcément tranché le morceau de la queue du lion que le serpent-dragon avait avalé. C'est à ce prix que le lion a été délivré de la gueule du grand prédateur. L'effet de cette opération est la domestication du lion qui s'exprime par des gestes anthropomorphes signifiant la gratitude et la soumission: il s'agenouille, tend les pattes jointes, verse des larmes. Ce devenir-humain du lion peut être interprété allégoriquement comme image du redevenir-humain du protagoniste qui, après avoir récupéré la raison et la condition de chevalier, est en mesure de faire un choix moralement fondé. Toutefois, le lion n'est pas une image symbolique ou, s'il en est une - une image de la re(auto)domestication d'Yvain -, sa fonction ne s'y réduit pas. Car le lion prend place à côté d'Yvain et plus jamais ne le quittera. L'amitié de l'homme et de l'animal se traduit dans la coopération en ce qui concerne tout d'abord l'alimentation. C'est le fauve qui chasse et la différenciation des styles humain et animal de se nourrir est assurée: le lion capture un chevreuil, en boit le sang, puis Yvain l'écorche, le démembre et le fait rôtir. Comme un chien, le lion veille le sommeil de son maître. Il y a une alternance entre des comportements de fauve et d'homme. Le lion est le miroir de l'homme surtout dans l'expression de la douleur qui va jusqu'à une tentative de suicide par l'épée, lorsqu'il croit à tort que son seigneur, qui s'était évanoui de douleur à la vue de la Fontaine, domaine de la Dame, est mort. Cette humanisation à outrance n'est-elle pas une façon de refuser à l'animal d'être ce qu'il est? L'anthropomorphisme du lion est si excessif que le pathétique de la scène tourne au comique et on a l'impression que le roman parodie la figure du speculum typique des bestiaires.

Mais entre le héros et l'animal s'établissent des formes de coopération qui assument les différences entre les espèces. Le lion participe aux combats d'Yvain. Lorsqu'il voit son seigneur en danger, il intervient. Il arrache un gros morceau de la hanche du géant Harpin de la Montagne, être à demi-humain à demi-animal (immense, velu, couvert d'une peau d'ours), puis détache son épaule. Comme une partie de chasse, le combat se clôt en curée: "Car tuit keurent a la cuirie/ si com li chien qui ont chacie/ la venison tant qu'ils l'ont prinze" (vv. 4245-47). Les interventions de la bête introduisent la prédation dans le combat et la chevalerie devient un exercice simultanément humain et animal. Est-ce là une façon de suggérer les racines cynégétiques de l'activité militaire, l'indiscernabilité du combat et de la chasse? 
La présence symbolique du lion dans le (sur)nom du chevalier - un nom qui en fait est un masque -, tout en exprimant sa présence réelle auprès d'Yvain, porte sans doute des significations morales: la force, le courage, la générosité, la fidélité, la sollicitude, la noblesse, la qualité solaire du lion se trouvent chez le chevalier. Le chevalier au lion (qui a un lion) est un chevalier-lion (qui est un lion, qui possède ses qualités). Mais l'existence du lion n'est pas que nominale, elle est aussi et surtout réelle, le verbe être n'absorbant pas le verbe avoir, la métaphore n'assimilant pas la métonymie. Le lion est une bête de chair et de sang dont le corps sort des affrontements tout blessé. Après le combat contre les trois accusateurs de Lunete, le lion est si mal en point qu'Yvain le couche dans l'envers de son écu et c'est lui-même qui transporte ainsi son fidèle compagnon souffrant. "Perhaps the best semiotic trick that Chrétien plays occurs when le chevalier au lion arrives at a castle bearing a shield which does not have the image of a lion painted on it, but bearing, rather, the real lion, which has been wounded in combat"28. C'est dire que, en consonance avec son surnom, l'écu d'Yvain porte un lion, non pas sous la forme stylisée et épurée d'un blason ou armoirie, mais sous le poids d'un être vivant au corps meurtri. Le lion est bien le signe héraldique du chevalier, mais c'est un signe qui saigne.

Conclusion

Cette héraldique vivante n'autorise pas la réduction du lion réel à un symbole. L'héraldique du Chevalier au Lion garde la tension entre l'épaisseur de la substance vivante et l'extrême minceur du signe, ce qui est une stratégie de relativisation voire d'oblitération de la pensée symbolique. L'anthropomorphisme des bestiaires est par ailleurs parodié. La figuration de la relation humain-animal dans ce roman indique une distance critique considérable de la part de Chrétien par rapport aux mécanismes analogiques de la pensée symbolique. On dirait que, pour lui, ces mécanismes ne rendent pas suffisamment compte de la complexité des relations qu'humains et animaux peuvent entretenir. Le compagnonnage du chevalier et du lion, qui se matérialise dans la réciprocité des soins, coexiste avec le rapport hiérarchique établi par et avec le geste tranchant qui différentie et domestique. Et pourtant, la domestication n'a pas privé le lion de sa férocité de fauve, elle l'a juste canalisée vers les ennemis de son maître, en en faisant ainsi un instrument de sa soumission à l'homme. La férocité du lion n'est pas arbitraire ou simplement instinctive, mais obéit à un critère dicté par l'intérêt de l'homme. L'anthropocentrisme de Chrétien n'est pas de nature théologique et ne mobilise la métaphore du speculum que par la sémiose dissociative de l'ironie ${ }^{29}$ qui fragilise ou suspend la pensée symbolique. Il apparait au sein d'un récit qui interroge l'animalité humaine ${ }^{30}$ en aménageant des zones liminaires qui renvoient ou évoquent confusément des phases de l'anthropogenèse et où se mettent en place des formes inusitées de coopération et d'amitié entre un homme et un animal. Cette relation qui est simultanément de maîtrise et de réciprocité, appelle à une approche zoopoétique qui valorise la complexité de la représentation du lien hommeanimal dans ce roman et les significations porteuses de vie qui se dégagent de son anthropocentrisme ou de son humanisme ${ }^{31}$. Il ne faut pas oublier que les trois figures qui thématisent l'animalité humaine - le bouvier, le fou et le chevalier au lion s'insèrent dans un roman courtois dont le sujet majeur est l'amour. $\mathrm{Si}$, comme le dit Agamben, l'aventure est un processus de subjectivation du vivant dans et par l'amour, si l'amour est l'expérience ontologique décisive qui ébranle les frontières humaines, 
alors Le chevalier au lion pose le rôle qu'y joue le lien à un animal. Avec le lion, Yvain fait l'expérience continue de la sollicitude infinie qu'il étend aux personnages à qui il porte secours (Lunete, les tisseuses de Pesme Aventure, la sœur de Noire Épine, entre autres). L'empressement à aider les autres, à se mettre à leur service, n'est-ce pas là l'assomption d'une discipline qui domestique et régule la passion, cette passion qui avait causé sa folie et sa sauvagerie, qui l'avait dédomestiqué et réanimalisé? Ce qu'Yvain apprend avec le lion n'est-ce pas à sublimer la passion, ou la pulsion, à la réorganiser socialement sous forme de la sollicitude avant et afin de retrouver l'amour de la Dame? ${ }^{32}$ C'est dire que le lion joue un rôle clé dans l'apprentissage de l'amour courtois entendu comme sublimation de la pulsion sexuelle en lien social via la discipline de l'Éros (au sens platonique du terme) qui indexe l'aspiration au bonheur aux idéaux altruistes. La fonction du lion est donc nettement humaniste.

\section{BIBLIOGRAPHIE}

Sources

CHRÉTIEN DE TROYES - Le Chevalier au Lion. Édition critique d'après le manuscrit B.N. fr.1433, traduction, présentation et notes de David F. Hult. Paris: Librairie Générale Française, 1994.

Études

AGAMBEN, Giorgio - L'aventure. Paris: Rivages, 2016.

ÁLVARES, Cristina; DIOGO, Américo - "La demoiselle de Norison: la fin d'un monde où l'on croyait aux fées”. Sénéfiance 33 (1993), pp. 9-22.

BARATAY, Eric - “L'homme et l'animal. Quelle histoire?". Le paratonnerre [en ligne] (2017) [consulté le 30 novembre 2019]. Disponible à https://leparatonnerre.fr/2017/06/14/lhomme-etlanimal-quelle-histoire/.

BARTHOLEYNS, Gil; DITTMAR, Pierre-Olivier; Jolivet, Vincent - Image et transgression au Moyen Âge. Paris: PUF, 2008.

BIANCIOTTO, Gabriel (trad.) - Bestiaires du Moyen Âge. Paris: Stock, 1980.

CARRETO, Carlos - "Global Middle Ages ou as virtudes do anacronismo. A lição do texto medieval". E-letras com vida [en ligne] 2 (2019), pp. 118-149. [Consulté le 30 novembre 2019]. Disponible à https://e-lcv.online/index.php/revista/article/view/66

DESCOLA, Philippe - Par-delà nature et culture. Paris: Gallimard. 2005.

DITTMAR, Pierre-Olivier - “Le devenir sans l'animal”. Images re-vues 6 (2009), pp. 1-5.

DOUDET, Estelle - "Le Chevalier au Lion: orgueil et charité". Acta Litt\&Arts [en ligne] 6 (2018).

[Consulté le $1^{\text {er }}$ décembre 2019]. Disponible à http://ouvroir-litt-arts.univ-grenoble-alpes.fr/ revues/actalittarts/359-le-chevalier-au-lion-orgueil-et-charite

DUFOURNET, Jean - "Le lion d'Yvain”. in Le Chevalier au Lion. Approches d'un chef-d'œuvre. Paris: Champion, 1988, pp. 77-104. 
FRAPPIER, Jean - Étude sur Yvain ou Le chevalier au lion de Chrétien de Troyes. Paris: SEDES, 1969.

GUIETTE, Robert - "Symbolisme et sénéfiance au Moyen Âge”. Cahiers de l'Association internationale d'études françaises 6 (1954), pp. 107-122.

HAIDU, Peter - Lion queue-coupée. L'écart symbolique chez Chrétien de Troyes. Genève: Droz, 1972.

HAIDU, Peter - "La sémiose dissociative. La signification historique du phénomène stylistique ‘Chrétien de Troyes' en France du Nord au XIIe siècle”. Europe 642 (1982), pp. 36-47.

HAIDU, Peter - “The Hermit's Pottage. Deconstruction and History in Yvain”. Romanic Review 74 (1983), pp. 1-15.

LE GOFF, Jacques; VIDAL-NAQUET, Pierre - “Lévi-Strauss en Brocéliande”. Critique 325 (1974), pp. 541-574.

LEUPIN, Alexandre - Fiction et incarnation. Littérature et théologie au Moyen Âge. Paris: Flammarion, 1993.

MOSCOVICI, Serge - La société contre nature. Paris: UGE, 1972.

PASTOUREAU, Michel - Figures et couleurs. Etudes sur la symbolique et la sensibilité médiévales. Paris: Le Léopard d'or, 1986.

PASTOUREAU, Michel - Une histoire symbolique du Moyen Âge occidental. Paris: Seuil, 2004.

SIMON, Anne - "Une arche d'études et de bêtes". Revue des Sciences Humaine. Zoopoétique 328 (2017), p. 7-16.

SLOTERDIJK, Peter - Bulles. Sphères I. Paris: Fayard, 2011.

STRUBEL, Armand - "Littérature et pensée symbolique au moyen âge (peut-on échapper au "symbolisme médiéval"?)". in BOUTET, Dominique et HARF-LANCNER, Laurence (eds.) - Écriture et modes de pensée au Moyen âge (VIIIe-XVe siècles). Paris: Presses de l'ENS, 1993, pp. 27-45.

VANCE, Eugene - "From Man-Beast to Lion-Knight". in From Topic to Tale. Logic and Narrativity in the Middle Ages. Minneapolis: University of Minnesota Press, 1987, pp. 80-108.

VANCE, Eugene - From Topic to Tale. Logic and Narrativity in the Middle Ages. Minneapolis: University of Minnesota Press, 1987.

VANCE, Eugene - "Si est homo, est animal". in From Topic to Tale. Logic and Narrativity in the Middle Ages. Minneapolis: University of Minnesota Press, 1987, pp. 53-79.

WALTER, Philippe - Canicule. Essai de mythologie sur Yvain de Chrétien de Troyes. Paris: SEDES, 1988.

\section{NOTES}

1. DESCOLA, Philippe - Par-delà nature et culture. Paris: Gallimard. 2005. Selon Descola, le concept majeur de l'analogisme est la chaîne ou échelle des êtres qui est l'organisation hiérarchique du vivant.

2. Sur la pensée symbolique médiévale lire GUIETTE, Robert - "Symbolisme et sénéfiance au Moyen Âge". Cahiers de l'Association internationale d'études françaises 6 (1954), pp. 107-122; PASTOUREAU, Michel - Figures et couleurs. Etudes sur la symbolique et la sensibilité médiévales. Paris: Le Léopard d'or, 1986; PASTOUREAU, Michel - Une histoire symbolique du Moyen Âge occidental. Paris: Seuil, 2004. En ce qui concerne spécifiquement 
Le chevalier au Lion, on citera l'étude de symbolique astrologique de WALTER, Philippe Canicule. Essai de mythologie sur Yvain de Chrétien de Troyes. Paris: SEDES, 1988.

3. DITTMAR, Pierre-Olivier - “Le devenir sans l'animal". Images re-vues 6 (2009), p.2.

4. BARTHOLEYNS, Gil; DITTMAR, Pierre-Olivier; Jolivet, Vincent - Image et transgression au Moyen Âge. Paris: PUF, 2008.

5. CARRETO, Carlos - "Global Middle Ages ou as virtudes do anacronismo. A lição do texto medieval”. E-letras com vida [en ligne] 2 (2019), pp. 118-149. [Consulté le 30 novembre 2019]. Disponible à https://e-lcv.online/index.php/revista/article/view/66.

6. CARRETO, Carlos - "Global Middle Ages ou as virtudes do anacronismo. A lição do texto medieval" ..., pp. 122-129.

7. Sur cette question, lire l'excellent dossier "Lire et écrire au Moyen Âge" dans la revue L'Histoire 463 (2019) pp. 35-63.

8. SLOTERDIJK, Peter - Bulles. Sphères I. Paris: Fayard, 2011, pp. 74-75.

9. LEUPIN, Alexandre - Fiction et incarnation. Littérature et théologie au Moyen Âge. Paris: Flammarion, 1993.

10. VANCE, Eugene - From topic to tale ..., p. 53.

11. Il y a presque une trentaine d'années, Armand Strubel avait problématisé l'idée de la littérature comme lieu de dévoilement de la pensée symbolique et mis en question la pertinence de la lecture symbolique des textes littéraires. Sa conclusion est que s'il y a une vision du monde symbolique spécifiquement médiévale, "la littérature n'en participe que de façon marginale, accessoire et occasionnelle". STRUBEL, Armand - "Littérature et pensée symbolique au moyen âge (peut-on échapper au "symbolisme médiéval"?)". in BOUTET, Dominique et HARF-LANCNER, Laurence (eds.) - Écriture et modes de pensée au Moyen âge (VIIIe-XVe siècles). Paris: Presses de l'ENS, 1993, pp. 27-45.

12. LEUPIN, Alexandre - Fiction et Incarnation....

13. BARATAY, Eric - “L'homme et l'animal. Quelle histoire?". Le paratonnerre [en ligne] (2017) [consulé le 30 novembre 2019]. Disponible à https://leparatonnerre.fr/ 2017/06/14/lhomme-et-lanimal-quelle-histoire/.

14. BIANCIOTTO, Gabriel (trad.) - Bestiaires du Moyen Âge. Paris: Stock, 1980.

15. SIMON, Anne - "Une arche d'études et de bêtes". Revue des Sciences Humaine. Zoopoétique 328 (2017), p. 9.

16. SIMON, Anne - "Une arche d'études et de bêtes"..., p. 13.

17. SIMON, Anne - "Une arche d'études et de bêtes" ..., p. 15.

18. Citons DUFOURNET, Jean - "Le lion d'Yvain". in Le Chevalier au Lion. Approches d'un chefd'œuvre. Paris: Champion, 1988, pp.77-104; FRAPPIER, Jean - Étude sur Yvain ou Le Chevalier au Lion de Chrétien de Troyes. Paris: SEDES, 1969, pp. 212-218.

19. Par exemple, HAIDU, Peter - Lion queue-coupée. L'écart symbolique chez Chrétien de Troyes. Genève: Droz, 1972, pp. 58-73; VANCE, Eugene - "From Man-Beast to Lion-Knight". in From Topic to Tale. Logic and Narrativity in the Middle Ages. Minneapolis: University of Minnesota Press, 1987, pp. 105-106.

20. SIMON, Anne - "Une arche d'études et de bêtes"..., p. 15.

21. SIMON, Anne - "Une arche d'études et de bêtes"..., p. 15.

22. AGAMBEN, Giorgio - L'aventure. Paris: Rivages, 2016, pp. 71-75.

23. C'est la thèse de l'hominisation comme cynégétisation soutenue par MOSCOVICI, Serge - La société contre nature. Paris: UGE, 1972.

24. LE GOFF, Jacques; VIDAL-NAQUET, Pierre - “Lévi-Strauss en Brocéliande”. Critique 325 (1974), p. 552.

25. Peter Haidu et Eugene Vance ont discuté la structure rhétorique de cet épisode ainsi que ses significations économiques, sociales et politiques: HAIDU, Peter - “The Hermit's 
Pottage. Deconstruction and History in Yvain". Romanic Review 74 (1983), pp. 1-15. VANCE, Eugene - "Si est homo, est animal". in From Topic to Tale. Logic and Narrativity in the Middle Ages. Minneapolis: University of Minnesota Press, 1987, pp. 68-79.

26. LE GOFF, Jacques; VIDAL-NAQUET, Pierre - "Lévi-Strauss en Brocéliande” ..., pp. 570-571.

27. ÁlVARES, Cristina; DIOGO, Américo - "La demoiselle de Norison: la fin d'un monde où l'on croyait aux fées". Sénéfiance 33 (1993), pp. 9-22.

28. VANCE, Eugene - "From Man-Beast to Lion-Knight" ..., p. 106.

29. HAIDU, Peter - "La sémiose dissociative. La signification historique du phénomène stylistique 'Chrétien de Troyes' en France du Nord au XIIe siècle”. Europe 642 (1982), p. 39.

30. Dans From Topic to Tale..., Eugene Vance soutient que Le chevalier au lion est le développement narratif d'un topos logique qui pose la relation d'un être humain à son animalité générique, et qu'Abélard a formulé dans la maxime: "si est homo, est animal" (VANCE, Eugene - Si est homo ..., pp. 53-54).

31. Vance considère Le chevalier au lion "as the very centerpiece of twelfth-century French humanism" (VANCE, Eugene - "From Man-Beast to Lion-Knight" ..., p. 108).

32. “(...) dans le roman courtois la fin'amor est la source et le suprême degré de la caritas universelle. Parce qu'il parvient à comprendre qu'il a trop vite assouvi un désir d'union qui aurait dû être le but de sa quête, Yvain transfigure ce qui était l'ultime étape de la passion érotique selon Richard de Saint-Victor, la haine de soi et la folie, en nouveau départ. Grâce aux aventures qui l'ont initié à l'élan compassionnel, le héros paraît capable - avec l'aide de la charitable Lunette - de mener son mariage au point où ce dernier se confond avec l'amour sacré" (DOUDET, Estelle - "Le Chevalier au Lion: orgueil et charité". Acta Litt\&Arts [en ligne] 6 (2018). [Consulté le $1^{\text {er }}$ décembre 2019]. Disponible à http://ouvroir-litt-arts.univ-grenoble-alpes.fr/revues/actalittarts/359-le-chevalierau-lion-orgueil-et-charite). Sauf pour l'identification de l'amour conjugal à l'amour sacré, qui nous semble excessive (et l'auteure le reconnait lorsqu'elle dit que le roman n'est pas un traité de théologie), nous souscrivons à cette affirmation en général.

\section{RÉSUMÉS}

Nous proposons une nouvelle lecture du Chevalier au Lion de Chrétien de Troyes inspirée de la zoopoétique, courant herméneutique théorisé par Anne Simon dans la mouvance de l'animal turn des sciences humaines et sociales. Centré sur la rencontre du chevalier Yvain et d'un lion, ce roman thématise l'animalité humaine et donne une configuration narrative à la relation humainanimal. Nous soutenons que cette configuration relève moins des procédés métaphoriques typiques de la pensée symbolique que de la contiguité (ou continuité) métonymique, laquelle favorise un imaginaire des liminalités, des passages et des échanges. Le Chevalier au Lion est un texte particulièrement propice à une approche zoopoétique pour autant que celle-ci cherche dans la littérature du passé des formes de figuration des animaux décalées par rapport à la tradition analogique de l'anthropomorphisme. Notre but est d'éclairer la modernité de l'humanisme de Chrétien de Troyes.

This paper proposes a new reading of Le Chevalier au Lion by Chrétien de Troyes inspired by the zoopoetics, a hermeneutical approach theorized by Anne Simon within the framework the animal 
turn in literary studies and human and social sciences. Centered on the meeting of the knight Yvain and a lion, this roman thematizes human animality and gives the human-animal relationship a narrative configuration. We argue that this configuration is a matter of metonymic contiguity (or continuity) rather than of metaphoric techniques typical of the symbolic thought, and that metonymic figuration favours a dynamic of liminalities, passages and exchanges. Le Chevalier au Lion is a text particularly fit for a zoopoetic approach as zoopoetics looks, in literary texts from the past, for ways of representing animals that challenge the analogic tradition of anthropomorphism. In short, the paper aims at shedding light on the modernity of Chrétien's humanism.

INDEX

Mots-clés : Humain, Animal, Zoopoétique, Pensée symbolique, Liminalités

Keywords : Human, Animal, Zoopoetics, Symbolic thought, Liminalities

\section{AUTEUR}

\section{CRISTINA ÁLVARES}

Universidade do Minho, Instituto de Letras e Ciências Humanas, Centro de Estudos Humanísticos (CEHUM). 4710-052 Braga, Portugal. calvares@ilch.uminho.pt . https://orcid.org/

0000-0001-5968-4724 